value for the enthalpy of adsorption of both $\mathrm{CO}$ and $\mathrm{NO}$.

A plausible explanation of the correlation observed is as follows. The standard entropy of adsorption is ascribable to the stretch of the cation-carbon (or cation-nitrogen) bond and to two bending modes of $\mathrm{CO}$ (or NO). The frequencies involved are usually (see, e.g., ref 19) assumed to parallel the strength of adsorption $\Delta_{a} H$. As a matter of fact, the Ni-N (or similar) motion can be represented by a Morse function, whose depth is the adsorption energy: hence the relationship between the cation-carbon (or nitrogen) stretching frequency and the strength of adsorption. As to the bending vibrations (deformations), there is little direct evidence. In a set of systems, however, not too far from that under study (namely $\mathrm{H}$-bonded complexes of the type FH-B, where $\mathrm{B}$ is a base molecule), the libration modes of HF have been observed to parallel the strength of adsorption. ${ }^{20}$ The entropy associated with a harmonic vibration is a decreasing function of the frequency of vibration. ${ }^{19}$ In conclusion, the greater the strength of adsorption, the smaller the entropy associated with the residual vibrations of the adsorbed molecules, as observed.

(19) Tompkins, F. C. Chemisorption of Gases on Metals; London, 1978; p 131.

(20) Andrews, L. J. J. Phys. Chem. 1984, 88, 2940.

\section{Conclusions}

Whereas the region $\theta>0.5$ requires ad hoc measurements for a satisfactory thermodynamic characterization, the data concerning the region $0<\theta \leq 0.5$ allow a number of conclusions to be drawn on the basis of simple pairwise models.

The repulsion between NN admolecules is too strong to allow the formation of NN pairs: this is in line with the experimental finding that adsorption on adjacent sites takes place (nearly) after the completion of the $c(2 \times 2)$ structure and implies tilting of the surrounding admolecules.

The decline in the molar enthalpy of adsorption is thus ascribable to NNN repulsions, the extent of which may be evaluated from the value at $\theta=0.5$.

Considering the adsorption as only occurring on the $c(2 \times 2)$ lattice, one can apply the Wang model (QC approach). The decline in the heat of adsorption is satisfactorily accounted for, in sharp contrast with the entropy of adsorption.

The standard entropy of adsorption increases, passing from $\theta \approx 0$ to $\theta=0.5$; this is in line with an already reported correlation between standard entropy and molar enthalpy of adsorption.

Registry No. NiO, 1313-99-1; NO, 10102-43-9.

\title{
Salt Effects in Free Nonionic Films
}

\author{
Boomgaard, Th. van den and J. Lyklema* \\ Wageningen Agricultural University, Department of Physical and Colloid Chemistry, \\ P.O. Box 8038, 6700 EK Wageningen, The Netherlands
}

Received June 17, 1988. In Final Form: October 14, 1988

\begin{abstract}
The thickness has been measured of macroscopic aqueous films, stabilized by nonionic surfactants consisting of a hydrophobic part and a poly(ethylene oxide) moiety. Special attention has been paid to the influence of electrolytes. In the films, the surfactants behave like coiled polymers. Addition of electrolytes increases the rate of drainage. As a function of the electrolyte concentration, the equilibrium thickness passes through a maximum. These maxima are similar to those for the Huggins constant for the corresponding micelles.
\end{abstract}

The interaction between electrolytes and nonionic surfactants continues to be topical, and there are several reasons for this interest. The widespread use of poly(oxyethylene) as a polymer and as the hydrophilic moiety in the most important class of nonionic surfactants is one of these reasons. Salt effects are particularly relevant when the chemicals named act as stabilizers for colloids. More to the basic side, the mode of interaction of ions with the oxyethylene group, and perhaps with other parts of the molecule, poses a number of interesting problems. The purpose of the present paper is to contribute to the insight into this matter by studying the influence of some electrolytes on the thickness of free aqueous films, stabilized by nonionics.

More particularly, the surfactant Synperonic NPE-1800 will be emphasized. Although this substance is a commercial product and not homodisperse, its solution ${ }^{1}$ and adsorption properties ${ }^{2}$ have been studied in some detail, ${ }^{3}$

\footnotetext{
${ }^{\dagger}$ Present address: Department of Chemical Engineering, University of Twente, The Netherlands.
}

Table I. Surfactants Used

\begin{tabular}{lcc}
\hline systematic name & commercial code & $M_{\mathrm{n}} \times 10^{-3}$ \\
\hline $\mathrm{C}_{9} \mathrm{PhP}_{(13)} \mathrm{E}_{\{26\rangle}$ & NPE-1800 & $2.15 \pm 0.02$ \\
$\mathrm{C}_{9} \mathrm{PhP}_{(13)} \mathrm{E}_{\langle 50\rangle}$ & NPE-A & $3.15 \pm 0.02$ \\
$\mathrm{C}_{9} \mathrm{PhP}_{(13)} \mathrm{E}_{\{85\rangle}$ & NPE-B & $4.73 \pm 0.03$ \\
$\mathrm{C}_{9} \mathrm{PhP}_{(13)} \mathrm{E}_{\langle 26\rangle}$ & NPE-C & $9.33 \pm 0.07$ \\
$\mathrm{C}_{9} \mathrm{PhE}_{\langle 10\rangle}$ & NP-10 & $0.66 \pm 0.01$ \\
$\mathrm{C}_{9} \mathrm{PhE}_{\langle 15\rangle}$ & NP-15 & $0.88 \pm 0.01$ \\
$\mathrm{C}_{9} \mathrm{PhE}_{(20)}$ & NP-20 & $1.10 \pm 0.01$
\end{tabular}

so they are well characterized. Comparison of the film results with those of the previus work is helpful to clarify and analyze trends. With regard to the electrolyte concentration, a large range of concentrations will be studied.

One of the main motivations is that the Huggins constant of NPE-1800 micelles passes through a maximum as

(1) Boomgaard, Th. van den; Zourab, Sh. M.; Lyklema, J. Progr Colloid Polym. Sci. 1983, 68, 25.

(2) Boomgaard, Th. van den; Tadros, Th. F.; Lyklema, J. J. Colloid Interface Sci. 1987, 116, 8 .

(3) Boomgaard, A. van den, Ph.D. Thesis, Wageningen Agricultural University, 1985. 


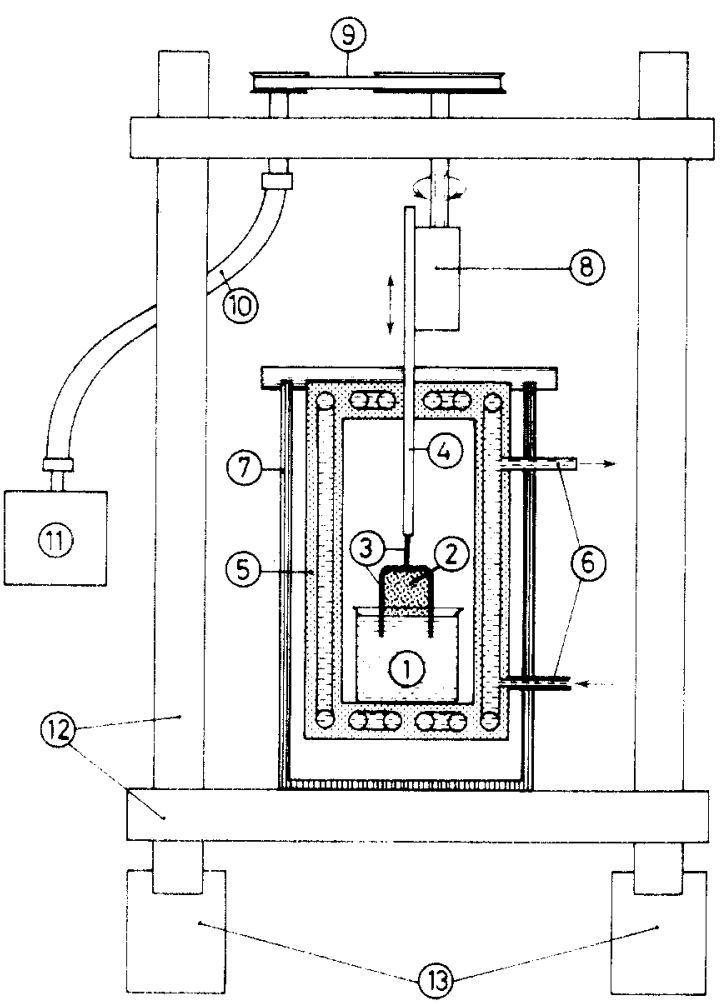

Figure 1. Schematic representation of the apparatus for measuring the thickness of free liquid films: (1) cuvette, containing surfactant solution, (2) film, (3) glass frame, (4) perspex rod, (5) water-thermostated brass box, (6) inlet and outlet for thermostating water, (7) perspex box, (8) device for raising or lowering the glass frame at controlled rate, (9) driving belt, (10) vibration-free flexible connection, (11) stepper motor, (12) metal support (part of optical bench), (13) vibration dampers.

a function of the concentrations of added $\mathrm{NaCl}, \mathrm{MgSO}_{4}$, or $\mathrm{Na}_{2} \mathrm{SO}_{4}{ }^{1}$ The maxima were situated at about $1.1,0.4$, and $0.2 \mathrm{M}$ ionic strength for these three salts, respectively, that is, at concentrations far above those where lyophobic sols would be coagulated by the mentioned electrolytes. An entirely satisfactory explanation has not yet been offered, although it may in this connection be recalled that electrolytes do exert their influence on adsorbed polyelectrolytes above the usual coagulation concentrations, a feature that has been theoretically predicted ${ }^{4}$ and experimentally observed. ${ }^{5}$ As NPE-1800 molecules adsorb as statistical coils, there may be some resemblance with polyelectrolytes adsorbed from salt solutions. However, these analogies require further substantiation, and a systematic study of the thickness of free aqueous films stabilized by NPE- 1800 is one means to obtain useful additional information.

\section{Experimental Section}

Materials. Most results to be reported have been obtained with Synperonic NPE-1800; a few additional measurements with other Synperonics have been included for the sake of comparison. Compositions and number-averaged relative molecular masses are collected in Table I. We refer to the surfactants using the same code as proposed by Clunie and Ingram ${ }^{6}$ (except for the abbreviation for phenyl), viz., $\mathrm{C}, \mathrm{Ph}, \mathrm{P}$, and $\mathrm{E}$ stand for the hydracarbon, phenyl, propylene oxide, and ethylene oxide moieties, respectively, with the subscripts giving the (average)

(4) Schee, H. A. van der; Lyklema, J. J. Phys. Chem. 1984, 88, 6661.

(5) Bonekamp, B. C.; Schee, H. A. van der; Lyklema, J. Croat. Chem. Acta $1983,56,695$.

(6) Clunie, J. S.; Ingram, B. T. In Adsorption from Solution at the Solid-Liquid Interface; Parfitt, G. D., Rochester, C. H., Eds.; Academic Press: New York, 1983; p 105.

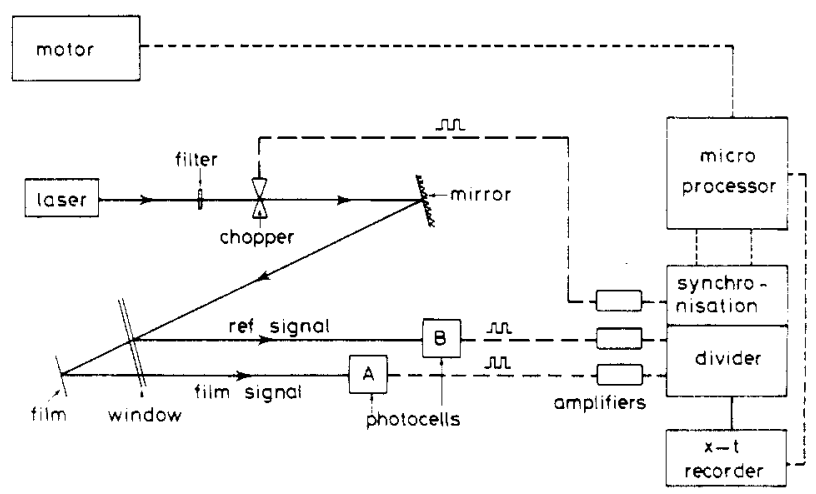

Figure 2. Block diagram of the optical and electronic parts of the apparatus for measuring film thicknesses.

lengths. Most of these surfactants were independently subjected to structural characterization, using PMR, chromatography, and UV spectroscopy. The hydrocarbon nonyl group is branched but probably always in the para position with respect to the poly(propylene oxide) or poly(ethylene oxide). The ethoxy part is heterodisperse and has a Poisson distribution. The colloid chemical properties of $\mathrm{C}_{9} \mathrm{PhP}{ }_{\langle 13\rangle} \mathrm{E}_{\langle 26}$ have been described before. ${ }^{1,3}$

The electrolytes were ex. Merck or ex. Baker and analytical grade. They were used without further purification.

Methods. We studied vertical, so-called macroscopic films, i.e., films with an area of a few square centimeters. The cell in which these films were studied is sketched in Figure 1 . It is a modification of an apparatus described in detail elsewhere. ${ }^{7}$ Films were formed in a glass frame, about $1.25 \mathrm{~cm}$ wide, $1.5 \mathrm{~cm}$ high, and $1.5 \mathrm{~mm}$ in cross section. Prior to each set of measurements with a given surfactant solution, the glass cuvette and frame were cleaned by immersing them in chromic acid for at least $12 \mathrm{~h}$, first rinsing several times with tap water and then with deionized water (Millipore Milli RO 60 filter, combined with a Super Q system, specific conductivity $<10^{-4} \Omega^{-1} \mathrm{~m}^{-1}$ ) followed by drying in air at $383 \mathrm{~K}$. Inside the brass box, the temperature was controlled to within $0.01 \mathrm{~K}$. In the front wall of this box, double glass windows were mounted.

The glass frame was attached to a vertical perspex rod, connected to a screw micrometer. It could be moved up and down at controllable speed, allowing several types of experiments to be done: thicknesses could be studied during the pull-out, during downward motion, or while stationary. Unless otherwise stated, all experiments reported in this paper have been performed by first pulling the frame out rapidly and then letting the film drain till equilibrium.

Care was taken to mount the whole system vibration free.

A block diagram of the optical and electronic part is given in Figure 2. Thicknesses were obtained from light reflection. The light source was a Spectra Physics Model $1550.5-\mathrm{mW} \mathrm{He}-\mathrm{Ne}$ laser with a characteristic wavelength $\lambda$ of $632 \mathrm{~nm}$. The beam was directed horizontally to the vertical film, meeting it at an angle of $7^{\circ}$ after attenuation (16×) and passing through a chopper and a mirror. The light spot on the film had a diameter of about 1 $\mathrm{mm}$ and was situated about $6 \mathrm{~mm}$ above the meniscus with the bulk solution. The beam reflected from the film was directed to a Hewlett Packard-PIN photodiode 5082-4204 photodetector (A in Figure 2). In order to eliminate fluctuation in the laser light intensity, use was made of a reference beam: light reflected by one of the glass windows was directed onto a second photodetector, identical to the first (see B in Figure 2). The two signals were electronically divided.

The drainage of a film, obtained by rapidly pulling out the frame, is recorded as a diagram in which the reflected intensity passes through a series of maxima almost exactly corresponding to thicknesses of $7 \lambda / 4,5 \lambda / 4,3 \lambda / 4$, and $\lambda / 4$, after which a constant value is attained, characterizing the equilibrium film. The elaboration of this signal to yield the equivalent water thickness $d_{w}$ (the thickness computed assuming the film to consist of pure water) and the conversion of $d_{\mathrm{w}}$ into the real film thickness $(d)$

(7) Lyklema, J.; Scholten, P. C.; Mysels, K. J. J. Phys. Chem. 1965 69,116 . 
Table II. Correction Parameters in the Conversion of the Equivalent Water Thickness $d_{w}$ into the Real Thickness ${ }^{a}$

\begin{tabular}{lccccccc}
\hline surfactants & $d_{1}$ & $\Delta d_{1}$ & $d_{2}$ & $\Delta d_{2}$ & $d_{3}$ & $\Delta d_{3}$ & $d-d_{\text {w }}$ \\
\hline NPE-1800 & 0.65 & -0.6 & 1.68 & -0.94 & 2.56 & -1.31 & -2.85 \\
NPE-A & 0.65 & -0.6 & 1.24 & -0.69 & 3.63 & -1.86 & -3.15 \\
NPE-B & 0.65 & -0.6 & 0.97 & -0.54 & 4.81 & -2.46 & -3.60 \\
NPE-C & 0.65 & -0.6 & 0.67 & -0.37 & 7.27 & -3.73 & -4.70 \\
NP-10 & 0.65 & -0.6 & & & 1.53 & -0.78 & -1.38 \\
NP-15 & 0.65 & -0.6 & & & 1.91 & -0.98 & -1.58 \\
NP-20 & 0.65 & -0.6 & & & 2.23 & -1.14 & -1.74
\end{tabular}

${ }^{a}$ Layers 1, 2 and 3 refer to the nonylphenol, poly(propylene oxide), and poly(ethylene oxide) fraction of the surfactants, respectively. All thicknesses in $\mathrm{nm}$.

have been described before..$^{7-9} \quad$ Basically, $d_{w}$ is obtained by comparing the intensity of the equilibrium film with that of the last maximum ( $\lambda / 4$ thickness), which usually can be accurately measured. Conversion of $d_{\mathrm{w}}$ into $d$ according to Frankel and Mysels $^{2}$ accounts for multiple reflection due to the internal inhomogeneity of the films; in this model, films are considered as multilayer systems, each layer having its specific index of refraction. Due to the oligomeric nature of our surfactants as reflected in their adsorption properties, ${ }^{2}$ the layers are not discrete but change their properties gradually in the direction normal to the film surface. However, Van Vliet ${ }^{10}$ has shown that replacement by a block distribution does not greatly affect the correction factor (about $10 \%$ of the correction, amounting to about $0.3 \mathrm{~nm}$ of uncertainty in our films). In our case, we used a seven-layer model for the correction of $d_{\mathrm{w}}$ for surfactants of the NPE series (NP, PPO, EO, water, EO, PPO, NP) and a five-layer model for the NP surfactants (NP, EO, water, EO, NP). Pertinent data are collected in Table II. The temperature was $298 \mathrm{~K}$ unless where stated otherwise.

Details on the choice of the correction parameters, as well as further information regarding the experimental part, have been given by Van den Boomgaard. ${ }^{3}$ The overall reproducibility of the obtained film thicknesses is better than $98 \%$.

\section{Results and Discussion}

General Features. All films were mobile according to the definition of Mysels, ${ }^{8}$ implying that they have a low surface shear viscosity. Concentrated electrolytes tend to increase the rate of drainage. For example, films of NPE-1800 drained in about $1.5 \mathrm{~h}$, but in $0.4 \mathrm{M} \mathrm{Na}_{2} \mathrm{SO}_{4}$ or $4 \mathrm{M} \mathrm{NaCl}$ this time was reduced to $0.5 \mathrm{~h}$. This is a surprising observation, since the addition of electrolytes markedly increases the viscosity of the mainly aqueous cores. Apparently, electrolytes lower the surface shear viscosity of the surfactants used by us. In turn, this conclusion implies (probably substantial) absorption of electrolytes by the adsorbed surfactant layers. It may be added that for well-damped films an even more pronounced drainage improvement by electrolytes has recently been found by Barneveld in our department, using welldefined, homodisperse surfactants of the $\mathrm{C}_{12} \mathrm{P}_{25}$ type.

Stable films could be obtained only at concentrations of at least 10 times $\mathrm{cmc}$. This observation may be related to the earlier finding that adsorption plateaus of these surfactants are also attained far above the $\mathrm{cmc}^{2}{ }^{2}$ in contrast to the situation with ionic surfactants. Further increase of the surfactant concentration, even by a factor of up to 500 , has no further effect on the thickness.

Effects of Ethylene Oxide Chain Length and Heterodispersity. In Table III film thicknesses for surfactants, differing with respect to the ethylene oxide chain length, are compared, both for the NPE and the NP series. In order to compare our data with those of other investigators, equivalent water thicknesses $\left(d_{\mathrm{w}}\right)$ are included;

(8) Mysels, K. J.; Shinoda, K.; Frankel, S. P. Soap Films. Studies of their Thinning and a Bibliography; Pergamon Press: New York, 1959. (9) Frankel, S. P.; Mysels, K. J. J. Appl. Phys. 1966, 37, 3725. 1.

(10) Viet, T. van Meded. Landbouwhogeschool Wageningen 1977, 77,
Table III. Equilibrium Thickness $d$ for Surfactants of Differing Ethylene Oxide Length ${ }^{a}$

\begin{tabular}{lccc}
\hline surfactant & $\left\langle n_{\mathrm{EO}}\right)$ & $d_{\mathrm{w}}, \mathrm{nm}$ & $d, \mathrm{~nm}$ \\
\hline NPE-1800 & 26 & 14.4 & 11.6 \\
NPE-A & 50 & 17.0 & 13.9 \\
NPE-B & 85 & 19.3 & 15.7 \\
NPE-C & 190 & 27.8 & 23.1 \\
NP-10 & 10 & 9.5 & 8.1 \\
NP-15 & 15 & 10.3 & 8.8 \\
NP-20 & 20 & 11.3 & 9.5
\end{tabular}

${ }^{a}$ No electrolyte added. $\left\langle n_{\mathrm{EO}}\right\rangle$ is the number-average number of EO segments in the molecule.

the difference between $d$ and $d_{\mathrm{w}}$ is given in the last column of Table II.

To our knowledge, no other data for exactly the same surfactants have been reported, but our thicknesses compare well with data obtained for similar surfactants. For instance, Bruil ${ }^{11}$ found $d_{\mathrm{w}}=9.6 \mathrm{~nm}$ for $\mathrm{C}_{8} \mathrm{PhE}_{\langle 11\rangle}$ and Paluch ${ }^{12}$ reported $d_{\mathrm{w}}=8.0 \mathrm{~nm}$ for $\mathrm{C}_{8} \mathrm{PhE}_{\langle 9\rangle}$. Both these data may be compared with our $d_{\mathrm{w}}=9.5$ for NP10.

In interpreting these thicknesses, it should be realized that they are probably determined by the steric interaction of the ethylene oxide moieties of the surfactants. In our preceding paper ${ }^{2}$ we collected evidence for behavior of these surfactants resembling that of adsorbed copolymers. In the adsorbed state at solid-liquid interfaces, the molecules are coillike, and this is probably also the case for molecules in free films. For adsorbed polymers, different thicknesses can be distinguished. The thickness observed in steric stabilization is closely related to the hydrodynamic thickness, ${ }^{13,14}$ which, in turn, is much greater that the ellipsometrically determined thickness. For films stabilized by PVA, this difference has been convincingly proven. ${ }^{15}$ The hydrodynamic layer thickness may be equated to the electrokinetically measured thickness provided the salt concentration is sufficiently low. ${ }^{14,16,17}$ For the surfactants of the NPE series, the indistinguishability of the electrokinetic and half the film thickness has been verified by us before. ${ }^{18}$

It would be interesting to compare our thicknesses with those obtained by direct force measurements between nonionic-covered mica surfaces. Such experiments have been recently reported by Claesson et al. ${ }^{19}$ and by Luck1.

(11) Bruil, H. G. Meded. Landbouwhogeschool Wageningen 1970, 70,

(12) Paluch, M. Polish J. Chem. 1980, 54, 1827.

(13) Scheutjens, J. M. H. M.; Fleer, G. J. Macromolecules 1985, 18, 1882.

(14) Koopal, L. K.; Hlady, V.; Lyklema, J. J. Colloid Interface Sci. $1988,121,49$.

(15) Lyklema, J.; Vliet, T. van. Faraday Discuss. Chem. Soc. 1978, 65, 25.

(16) Cohen Stuart, M. A.; Waaien, F. H. W. H.; Dukhin, S. S. Colloid Polym. Sci. 1984, 262, 423 .

(17) Cohen Stuart, M. A.; Mulder, J. W. Colloids Surf. 1985, 15, 49.

(18) Cohen Stuart, M. A.; Boomgaard, Th. van den; Zourab, Sh. M.; Lyklema, J. Colloids Surf. 1984, 9, 163.

(19) Claesson, P. M.; Kjellander, R.; Stenius, P.; Christenson, H. K. J. Chem. Soc. Faraday Trans. 1 1986, 82, 2735. 


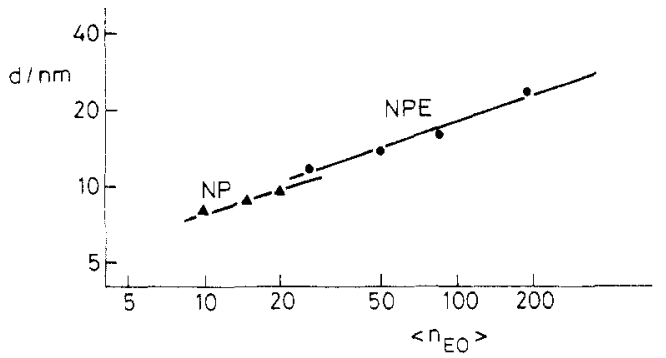

Figure 3. Double-logarithmic plot of the film thicknesses as a function of the average length of the ethylene oxide moiety.

ham and Klein. ${ }^{20}$ The former authors studied a homodisperse nonionic with a short ethylene oxide chain length $\left(C_{12} E_{5}\right)$. They found the repulsive force to increase rapidly (indicating steric repulsion) for interaction distances below about $3 \mathrm{~nm}$, depending on temperature. Luckham and Klein investigated Triton X-405, a heterodisperse nonionic with a long $\mathrm{EO}$ chain length, $\mathrm{C}_{8} \mathrm{PhE}_{\langle 11\rangle}$ in our notation. In their case, the pronounced rise of the force started below about $20 \mathrm{~nm}$. Broadly speaking, our results are between these two. It must be added that in heterodisperse systems a few large molecules may dominate the interaction; this feature tends to "swell" the films and may be (partly) responsible for the high interaction distances observed by Luckham and Klein. It must also be added that Luckham and Klein performed their measurements in $10^{-1} \mathrm{M} \mathrm{KNO}_{3}$, which may have affected the outcome somewhat.

The way in which $d$ depends on $\left\langle n_{\mathrm{EO}}\right\rangle$ gives some information on the mode of packing of the molecules in the film. $\left\langle n_{\mathrm{EO}}\right\rangle$ is the number-average number of EO segments in the molecule. By way of first approximation in Figure 3 a double-logarithmic plot of $d$ versus $\left\langle n_{\mathrm{EO}}\right\rangle$ is given. The two straight lines both have a slope of about 0.33 . The limited number of data points induces us not to overestimate this figure, but it may be inferred that the slope is not around 0.50 . The conclusion is that the coils are packed like statistical three-dimensional coils rather than like cylinders, in agreement with our finding concerning the adsorption of these surfactants at solid-liquid interfaces. ${ }^{2}$ Apparently, interaction does not grossly alter the conformation of the adsorbed molecules.

Over the range studied, the lines for NPE and NP (Figure 3) are parallel, but it is not likely that this parallelism persists for $\left\langle n_{\mathrm{EO}}\right\rangle \rightarrow 0$. Semiquantitatively, it may be inferred that films containing a polypropylene moiety are thicker by an amount which for $\left\langle n_{\mathrm{EO}}\right\rangle>20$ increases proportionally with $\left\langle n_{\mathrm{EO}}\right\rangle$. For $\left\langle n_{\mathrm{EO}}\right\rangle \rightarrow 0$ the contribution of the 13 polypropylene groups to $d$ is less than $1 \mathrm{~nm}$; i.e., they are fairly flatly packed.

The discussion of the thickness dependence upon 〈EO cannot be complete without considering the consequences of polydispersity. ${ }^{21}$ For polymers, the affinity for a surface increases rapidly with $M$. From a polydisperse mixture, the fractions with the higher $M$ adsorb preferentially. The extent to which this fractionation is effectuated in practice depends on the plateau range of the (adsorbent) area to (bulk) volume ratio, $A / V$. If this ratio is low, and if sufficient time is allowed for equilibration, a strong enrichment of the fractions with high $M$ takes place at the interface; in the opposite situation, little fractionation occurs.

As far as the adsorption of nonionic surfactants is concerned, some fractionation might also be anticipated, but not so strong as with polymers (because the affinity does

(20) Luckham, P. F; Klein, J. J. Colloid Interface Sci. 1987, 117, 149. (21) Cohen Stuart, M. A.; Scheutjens, J. M. H. M.; Fleer, G. J. J. Polym. Sci., Polym. Phys. Ed. 1980, 18, 559.

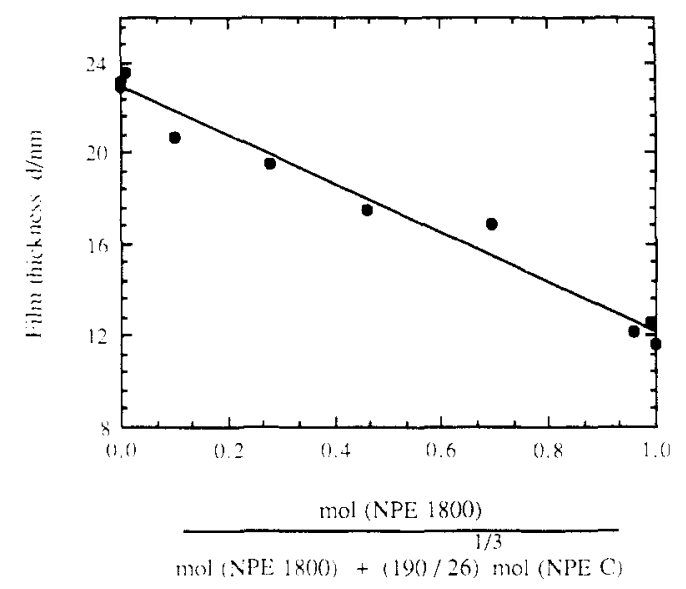

Figure 4. Film thickness as a function of composition in a mixture of Synperonics NPE-1800 and NPE-C. No electrolyte added. Temperature $298 \mathrm{~K}$.

not vary so strongly as a function of the EO length, which is not usually the anchoring moiety) and in the opposite direction (because the affinity decreases with increasing $\left.\left\langle n_{\mathrm{EO}}\right\rangle\right)$. For this reason, cross sectional areas in condensed monolayers, obtained at fluid/fluid interfaces from $\gamma$-ln $c$ measurements (low $A / V$ ) are not necessarily identical with those obtained from adsorption on solid surfaces, measured in disperse systems (high $A / V$ ). ${ }^{22}$ The extent to which fractionation could occur in thin films, depends on the method of preparation. In our technique and, for that matter, also in the usual procedures to make microscopic films, the area is fairly small, in contradistinction to methods involving foams.

In Figure 4, the thickness is given of films drawn from mixtures of NPE-1800 and NPE-C. In order to account for the difference in EO content between NPE-1800 and $\mathrm{NPC}$, on the abscissa the molar ratio contains a correction factor which equals the cubic root of the ratio of the $\mathrm{EO}$ contents between the two surfactants. The chosen power is based on the assumption of volume filling. However, if the power $1 / 2$ is chosen, no drastically different results are obtained. From this figure it can be concluded that there is no detectable preferential adsorption of one of the two surfactants. Our data are not compatible with the suggestion that the surfactants are adsorbed in a fully extended configuration, as has been inferred from direct measurement of the interaction force. ${ }^{20}$

Effect of Concentration and Nature of Electrolytes. Results for three different electrolytes are recorded in Figure 5. It is found that for all electrolytes the thickness as a function of $c_{\text {salt }}$ passes through a maximum.

As far as we are aware, such a maximum for film thicknesses of nonionics has not yet been reported in literature. Although in some publications salt effects have been studied, the results cannot be readily compared with ours because either the surfactant was very different or the range of electrolyte concentrations too small. A maximum as a function of $c_{\text {salt }}$ has been found by Clunie et $\mathrm{al}^{23}$ for films of dimethyldodecyl sulfoxide in $\mathrm{NaCl}$ (ionic strength $0-5 \mathrm{M}$ ) and by Buscall et al. ${ }^{24}$ for films stabilized by dodecyldiamine oxide in $\mathrm{KI}(0-2 \mathrm{M})$. In the latter case, there was also a $\mathrm{pH}$ dependence; hence the surfactant probably had some ionic character. The occurrence of the

(22) Aston, J. R. Ph.D. Thesis, University of Melbourne, 1987.

(23) Clunie, J. S.; Corkill, J. M.; Goodman, J. F.; Ingram, B. T. Special Discuss. Faraday Soc. 1970, 1, 30.

(24) Buscall, R.; Donaldson, R. B.; Ottewill, R. H.; Segal, D. In Foams Akers, R. J., Ed.; Academic Press: London, 1976; p 73. 


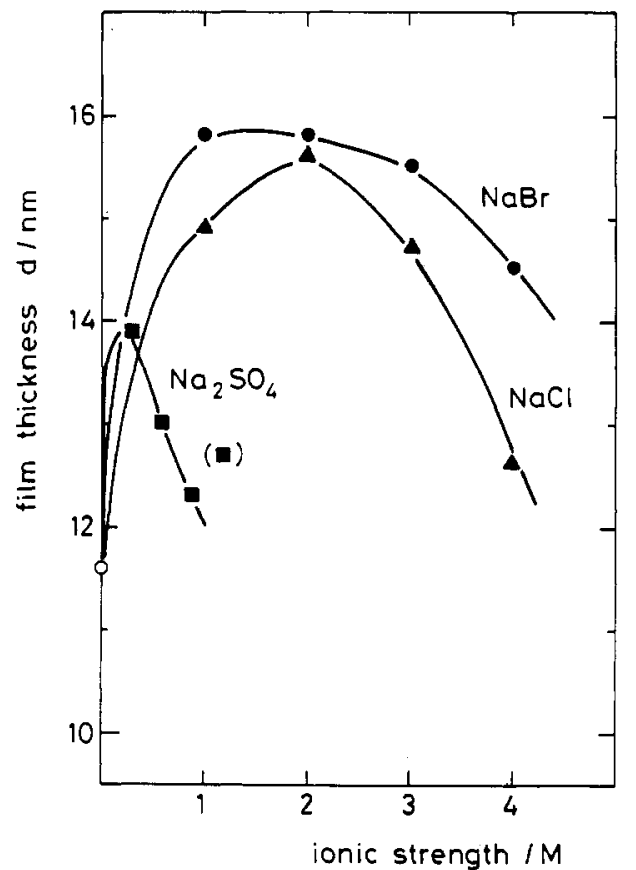

Figure 5. Influence of concentration and nature of three electrolytes on the thickness of films, stabilized by Synperonic NPE-1800.

maximum indicates that electrolytes exercise a dual influence, where it depends on the concentration and nature which of the two dominates. There is of course much literature on the influence of electrolytes on the cloud point of poly(ethylene oxides), and the trend that it decreases with increasing $c_{\text {salt }}$ is almost without exception. See the review by Durham ${ }^{25}$ and more recent examples. ${ }^{26,27}$ In other words, electrolytes lower the quality of water as a solvent for the polymer. If this were the only trend, the amount adsorbed should increase by the addition of electrolytes, because in poorer solvents longer loops are formed. However, the situation is more complex. Cloud points of nonionic surfactants on an ethylene oxide basis

(25) Durham, K. In Surface Activity and Detergency; Durham, K., Ed.; MacMillan: London, 1961; p 1.

(26) Boucher, E. A.; Hines, P. M. J. Polym. Sci., Polym. Phys, Ed. $1978,16,501$.

(27) Ataman, M. Colloid Polym. Sci. 1987, 265, 19. and critical flocculation temperatures of particles covered with such nonionics ${ }^{2}$ do not always decrease with $c_{\text {salt }}{ }^{25}$ Sometimes increases are observed over part of the concentration range. Perhaps it must be concluded that electrolytes do not only affect the conformation of the EO part of the molecule but also that of the hydrophobic fraction. This latter feature may be direct or indirect, in the sense that changes in the packing and conformation of the hydrophobic moiety may be dictated by the properties of the hydrophilic part. In this connection it is recalled that from their effect on the surface viscosity it was inferred that ions are probably absorbed inside the surfactant layer.

The most striking analogy is observed with the influence of electrolytes on the Huggins constant of NPE-1800 micelles, which also passes through a maximum as a function of $c_{\text {salt }}$ and where also the maximum for $\mathrm{Na}_{2} \mathrm{SO}_{4}$ was more pronounced than the maximum for $\mathrm{NaCl}^{1}{ }^{2}$ This observation confirms the experiments of Figure 4 and indicates that the hydrodynamic interaction between micelles (reflected in the film thickness) has similar roots. Apart from the confirmation of the similarity between the "hydrodynamic" and "steric" thickness of an adsorbed layer, ${ }^{18}$ the observed analogy indicates that the dual effect of electrolytes is independent of the geometry of the aggregate of the nonionic (flat or spherical).

We refrain from further speculation on the nature of the electrolyte effect. In our previous work ${ }^{1}$ it was suggested that interaction of salts with the poly(propylene oxide) fraction of the NPE Synperonics could play a role. Therefore, it would be of interest to repeat our measurements more systematically with surfactants without such a fraction. Provisional measurements performed by Barneveld in our department, using a very well-defined nonionic, confirmed the occurrence of the maximum. Hence, it appears that our finding is more general. In a later paper we intend to come back to this matter.

Acknowledgment. We thank Dr. Th. F. Tadros for useful discussions, Dr. R. I. Hancock of ICI-PLC Ltd. for providing the surfactants used, and Verhagen, Wegh, Keuskamp, van Beek, and Vos for technical help. The present study has been made possible by financial support from ICI.

Registry No. $\mathrm{NaCl}, 7647-14-5 ; \mathrm{MgSO}_{4}, 7487-88-9 ; \mathrm{Na}_{2} \mathrm{SO}_{4}$, 7757-82-6; synperionic NPE-1800, 9016-45-9. 